\title{
Clinical Effects and Complications of Pedicle Screw Augmentation with Bone Cement: Comparison of Fenestrated Screw Augmentation and Vertebroplasty Augmentation
}

\author{
Jin Hak Kim, MD, Dong Ki Ahn, MD, Won Shik Shin, MD, \\ Myung Jin Kim, MD, Ho Young Lee, MD, Young Rok Go, MD \\ Department of Orthopedic Surgery, Seoul Sacred Heart General Hospital, Seoul, Korea
}

Background: Pedicle screw augmentation with bone cement has been experimentally demonstrated to increase the pullout strength. However, the mechanisms of screw loosening are complicated and interacting. Although vertebroplasty augmentation and fenestrated screw augmentation have been compared in many studies, there has been no comparative study on their clinical effects and complications in real clinical settings. We investigated clinical effects of bone cement augmentation of a pedicle screw and differences according to augmentation methods.

Methods: Of the total 241 patients who had osteoporosis and underwent posterior pedicle screw fixation without anterior bone graft between January 2010 and December 2016, 132 patients with $\geq 2$ years of radiological follow-up were included in this retrospective study. The patients were divided into group I (unaugmented) and group II (bone cement augmented). Group II was subdivided into II-S group (solid screw augmented) and II-F group (fenestrated screw augmented). The incidence of screw loosening was compared between groups I and II. Cement leakage, screw loosening, and screw fractures were investigated in the subgroups.

Results: In total, 36 of 71 (52\%, group I) unaugmented cases and 96 of 170 (56\%, group II) augmented cases were followed up for $\geq 2$ years. Of the total 78 solid screw augmented cases, 42 (56\%) were in II-S group; 54 of the total $92(59 \%)$ fenestrated screw augmented cases were in II-F group. Groups I and II were homogenous regarding demographic characteristics; II-S and II-F groups were also homogenous. The incidence of screw loosening was $50.0 \%$ (18/36) in group I and 7.3\% (7/96) in group II ( $p<0.001)$. Cement leakage developed in 2 of 42 (4.8\%) cases in II-S group and in 5 of $54(9.3 \%)$ cases in II-F group ( $p=0.462)$. Screw loosening developed in 6 of $42(14.3 \%)$ cases in II-S group and in 1 of 54 cases (1.9\%) in II-F group ( $p=0.041)$. Screw fracture developed in none of 42 cases in II-S group and in 3 of 54 cases $(5.6 \%)$ in II-F group ( $p=0.254)$.

Conclusions: In osteoporotic patients, bone cement augmentation of a pedicle screw decreased the incidence of screw loosening, and fenestrated screw augmentation was more effective than vertebroplasty augmentation.

Keywords: Pedicle screw augmentation, Bone cement, Vertebroplasty, Fenestrated screw

Received August 15, 2019; Accepted September 18, 2019

Correspondence to: Dong Ki Ahn, MD

Department of Orthopedic Surgery, Seoul Sacred Heart General Hospital,

259 Wangsan-ro, Dongdaemun-gu, Seoul 02488, Korea

Tel: +82-2-966-1616, Fax: +82-2-968-2394

E-mail: adk0208@hanmail.net
Spine surgery is increasing in frequency in the elderly population with osteoporosis along with the increased life expectancy. In addition to osteoporosis, deformities that demand rigid fixation are frequently observed in elderly patients. However, it is not easy to achieve enough fixation strength even with a pedicle screw system in such patients. Among various methods to enhance fixation strength of 
Kim et al. Pedicle Screw Augmentation with Bone Cement

Clinics in Orthopedic Surgery • Vol. 12, No. 2, $2020 \bullet$ www.ecios.org

a pedicle screw, bone cement polymethyl methacrylate (PMMA) augmentation was experimentally proved as the most efficacious option. ${ }^{1-8)}$ Most previous experimental studies examined only the pullout strength, ${ }^{1,9-15)}$ while screw loosening in real clinical settings involves far more complicated mechanisms. Besides, there has been no study that documents the threshold of force required to prevent screw loosening in various conditions that are affected by the degree of osteoporosis, magnitude and location of deformity, and lifestyle. Though there were a few studies that proved clinical efficacy of PMMA augmentation, there has never been a study that compared it with a conventional method. In this study, we investigated the clinical effects of PMMA augmentation of pedicle screws especially on screw loosening. There are two different methods for PMMA augmentation: filling of PMMA with vertebroplasty followed by screw insertion; and PMMA augmentation through a fenestrated screw. We also assessed differences between the two methods in terms of their efficacy and complications.

\section{METHODS}

This is a retrospective observational study. We conducted this study in compliance with the principles of the Declaration of Helsinki. Written informed consents were obtained from all subjects. We reviewed patients who underwent posterior pedicle screw fixation in the thoracolumbar spine without anterior bone graft or cage insertion at our institution between January 2010 and December 2016. Of the total 241 patients, 132 patients with proper medical records and more than 2 years of radiological follow-up after surgery were included in this study. Patients who had an operation with conventional solid screws were classified as group I and those with PMMA augmentation as group II. Among group II, those who had prefilling of PMMA with vertebroplasty and solid screw insertion were categorized as II-S group and those who had PMMA augmentation through a fenestrated screw were categorized as II-F group. Before February 2014, all operations were performed by using the II-S method; the II-F method has been used thereafter. In the II-S group, augmentation was performed as follows: using a $2.8-\mathrm{mm}$-diameter cement injector containing $1.8 \mathrm{~mL}$ of PMMA, $1.8-3.6 \mathrm{~mL}$ of PMMA was instilled for each screw augmentation. Next, a solid screw was inserted at least 30 minutes after the prefilling of PMMA. In the II-F group, augmentation was performed as follows: a fenestrated screw that has a $2.5-\mathrm{mm}$ cannula and four holes at distal $1 / 3$ of the shaft was inserted and PMMA was instilled through an injector that has a nozzle and threads that interlock with the screw head (Fig. 1). Using an II-F injector containing $1.5 \mathrm{~mL}$ of PMMA, 1.5-3.0 $\mathrm{mL}$ of PMMA was instilled for each screw augmentation. High-viscosity PMMA (DePuy International Ltd., Leeds, UK) was used in all cases. The amount of instilled PMMA was determined discretionally according to the location of a segment, size of a vertebral body, and shape of distribution and leakage. Usually all screws were augmented, but in some cases, only screws at both ends were augmented. To verify the homogeneity between groups, demographic data and spine bone density were analyzed. Dual-energy $\mathrm{X}$-ray absorptiometry was used and accepted the lowest average T-score of at least more than two vertebrae. The primary endpoint was the difference in screw loosening between groups. In the subgroup analysis, leakage of PMMA (early complication) and screw loosening and breakage (late complications) were compared between the II-S group and II-F group. Screw loosening was defined as displacement of a screw from the original position or hollowness around a screw in a simple radiograph. If any of screws were loosened, the case was counted as screw loosening. The interpretation of radiological findings was done by two orthopedic surgeons (DKA and WSS). If there was disagreement, the final status was decided by a discussion between them.

Primary endpoint and subgroup analyses were done using the Fisher exact test. Intergroup homogeneity was assessed by the Student $t$-test for the parametric variables and by the Fisher exact test for nonparametric variable. SPSS ver. 16.0 (SPSS Inc., Chicago, IL, USA) was used for statistical analyses.

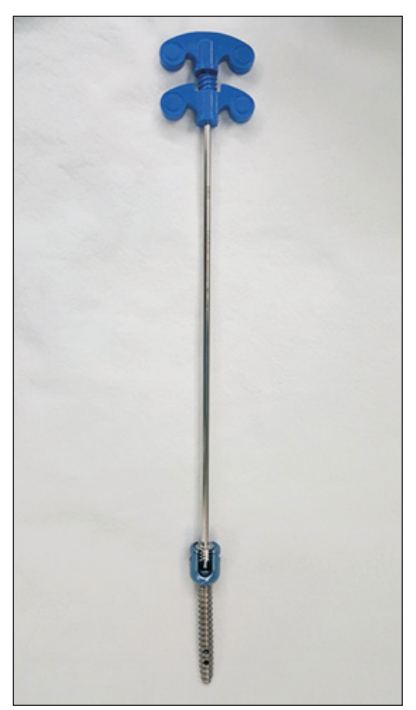

Fig. 1. Bone cement injector having a nozzle and threads that interlock with the canula and screw head. 
Kim et al. Pedicle Screw Augmentation with Bone Cement

Clinics in Orthopedic Surgery • Vol. 12, No. 2, 2020 • www.ecios.org

\section{RESULTS}

In total, 36 of the 71 (52\%, group I) unaugmented cases and 96 of the 170 (56\%, group II) augmented cases were followed up for more than 2 years. Of the total 78 solid screw augmented cases, 42 cases (56\%) were included in the subgroup II-S; of the total 92 fenestrated screw augmented cases, 54 cases (59\%) were included in the subgroup II-F (Fig. 2).

Group I and group II were homogenous in age (74.2 and 73.0 years, respectively), in sex (men : women, $10: 26$ and $18: 78$, respectively) and in spine bone density (T-score; -2.2 and -2.3 , respectively). II-S group and II-F group were homogenous in age (71.9 and 74.0 years, respectively), in sex (men : women, $6: 36$ and $12: 42$, respectively) and in spine bone density (T-score; -2.4 and -2.3 , respectively) (Table 1 ). The diagnoses of all patients are presented in Table 2.

The incidence of screw loosening was significantly

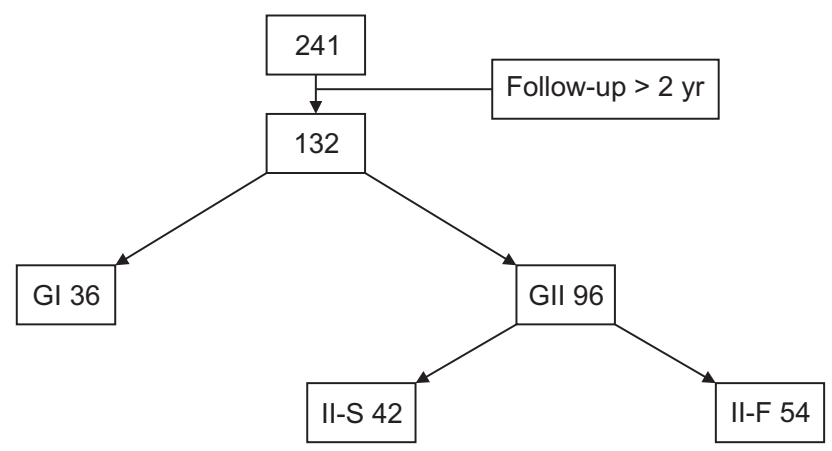

Fig. 2. Flowchart of study population selection. Gl: unaugmented group, GIl: bone cement augmented group, II-S: solid screw augmented group, II-F: fenestrated screw augmented group.

\section{Table 1. Demographic Data}

\begin{tabular}{lccc}
\hline \multicolumn{1}{c}{ Group } & Age (yr) & $\begin{array}{r}\text { Sex (male : } \\
\text { female) }\end{array}$ & $\begin{array}{c}\text { Bone mineral } \\
\text { density (T-score) }\end{array}$ \\
\hline I & $74.2 \pm 5.7$ & $10: 26$ & $-2.2 \pm 1.1$ \\
\hline II & $73.0 \pm 6.2$ & $18: 78$ & $-2.3 \pm 1.5$ \\
\hline II-S & $71.9 \pm 6.5$ & $6: 36$ & $-2.4 \pm 1.9$ \\
II-F & $74.0 \pm 5.9$ & $12: 42$ & $-2.3 \pm 1.3$ \\
\hline$p$-value (I vs. II) & $0.333^{*}$ & $0.339^{\dagger}$ & $0.758^{*}$ \\
\hline$p$-value (II-S vs. II-F) & $0.105^{*}$ & $0.431^{\dagger}$ & $0.798^{*}$ \\
\hline
\end{tabular}

Values are presented as mean \pm standard deviation. Group I: unaugmented, group II: bone cement augmented, group II-S: solid screw augmented, group II-F: fenestrated screw augmented.

${ }^{*} t$-test. ${ }^{\dagger}$ Fisher exact test. higher in group I (50.0\%; 18 in 36 patients) than in group II $(7.3 \%$; seven in 95 patients) $(p<0.001)$. On the subgroup analysis, leakage of PMMA developed in 2 of 42 patients $(4.8 \%)$ in the II-S group and in 5 of 54 patients $(9.3 \%)$ in the II-F group, showing no significant difference $(p=0.462)$. The two cases of cement leakage in the II-S group developed in normal vertebrae, whereas the leakage in the II-F group developed in fractured vertebrae in four cases and in a normal vertebra in one case; however, the difference was not statistically significant $(p=0.143$ ) (Table 3). PMMA leaked into the disc space in all of the two cases in the II-S group, whereas it leaked into the disc space in three cases and into the spinal canal in two cases in the II-F group. There was no complication associated with PMMA leakage. Screw loosening developed in 6 of 42 patients (14.3\%) in the II-S group and 1 of 54 patients $(1.9 \%)$ in the II-F group, showing significant difference $(p=0.041)$. None of the 40 patients in the II-S group had a screw fracture, whereas 3 of the 54 patients (5.6\%) in the II-F group had screw fractures; however, the difference was not statistically significant $(p=0.254)$ (Table 4$)$.

\begin{tabular}{|c|c|c|c|}
\hline \multirow{2}{*}{ Diagnosis } & \multicolumn{3}{|c|}{ Group } \\
\hline & 1 & II-S & $\|-F$ \\
\hline Fracture & 9 & 0 & 10 \\
\hline Traumatic myelopathy & 9 & 12 & 17 \\
\hline Traumatic kyphosis & 4 & 10 & 6 \\
\hline Traumatic stenosis & 7 & 14 & 12 \\
\hline Stenosis with deformity & 4 & 4 & 6 \\
\hline Others & 0 & 2 & 4 \\
\hline
\end{tabular}

Group I: unaugmented, group II: bone cement augmented, group II-S: solid screw augmented, group II-F: fenestrated screw augmented.

\begin{tabular}{|c|c|c|c|c|c|}
\hline \multirow{2}{*}{ Group } & \multirow{2}{*}{$\begin{array}{l}\text { Leakage } \\
\qquad(-)\end{array}$} & \multicolumn{3}{|c|}{ Leakage (+) } & \multirow{2}{*}{$p$-value* } \\
\hline & & Fractured V & Normal V & Total & \\
\hline II-S & 40 & 0 & 2 & 2 & 0.143 \\
\hline$\|-F$ & 49 & 4 & 1 & 5 & \\
\hline$p$-value* & & & 0.462 & & \\
\hline
\end{tabular}

Group II: bone cement augmented, group II-S: solid screw augmented, group II-F: fenestrated screw augmented.

$\checkmark$ : vertebra.

*Fisher exact test. 
Kim et al. Pedicle Screw Augmentation with Bone Cement

Clinics in Orthopedic Surgery • Vol. 12, No. 2, $2020 \bullet$ www.ecios.org

Table 4. Hardware Failure in Group II

\begin{tabular}{lccccc} 
& \multicolumn{5}{c}{ Screw failure } \\
\cline { 2 - 5 } Group & \multicolumn{2}{c}{ Loosening } & \multicolumn{2}{c}{ Fracture } \\
\cline { 2 - 6 } & + & - & + & - \\
II-S & 6 & 36 & 0 & 42 \\
II-F & 1 & 53 & 3 & 51 \\
p-value* & & 0.041 & & 0.254 \\
\hline
\end{tabular}

Group II: bone cement augmented, group II-S: solid screw augmented, group II-F: fenestrated screw augmented.

*Fisher exact test $(p<0.05)$.

\section{DISCUSSION}

Pedicle screw systems have very rigid fixation strength because they fix a vertebral segment, the strongest portion of the vertebral body, with a pedicle. However, loss of fixation is very common in elderly osteoporotic populations. $\left.{ }^{16}\right)$ Although stress at each pedicle screw can be reduced by wide distribution through long-segment fixation, this solution causes unnecessary stiffness and can result in fractures in adjacent vertebrae. To achieve stability with shortsegment fixation, purchase power of each screw should be enhanced. PMMA augmentation of a pedicle screw was proved to increase fixation strength in many experimental studies. While most of such studies documented that PMMA augmentation enhanced pullout strength, ${ }^{1,9-15)}$ the mechanism of screw loosening is a complex interaction of various loading patterns in vivo. ${ }^{10)}$ Furthermore, lifestyle, muscle strength, and sagittal or coronal balance may also be associated. Thus, the application of PMMA augmentation would bring about unexpected situations in vivo, which we assumed to be different according to the augmentation method in this study.

In the current study, the analysis of results was based not on each screw but on each patient because it was a clinical observational study. As the results showed, PMMA augmentation effectively reduced screw loosening without significant side effects. We also compared the two different PMMA augmentation methods. Until February 2014, we used the II-S method for PMMA augmentation. But it required a long wait time for the cure of PMMA before screw insertion and we experienced several episodes of failure. So, we changed to apply the II-F method thereafter. Although there have been many experimental studies that compare the pullout strength of the two methods, their results are conflicting: the II-S method was superior in two studies by Chen et al. ${ }^{11)}$ and Chang et

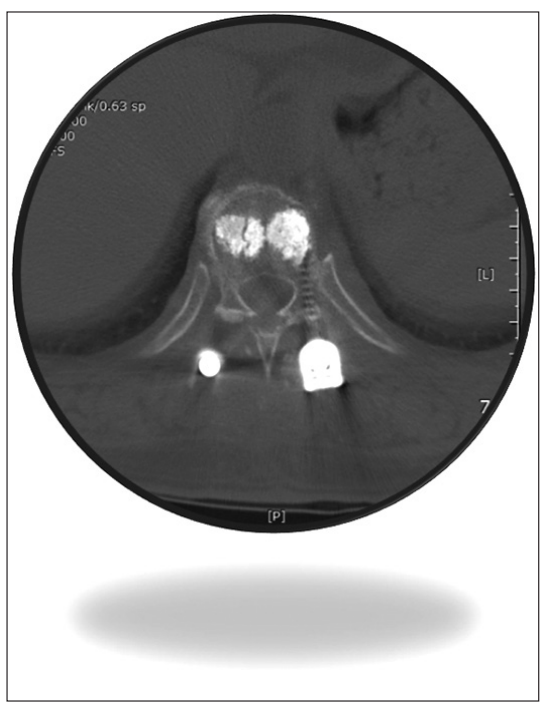

Fig. 3. Bone cement crack developed while inserting a solid screw.

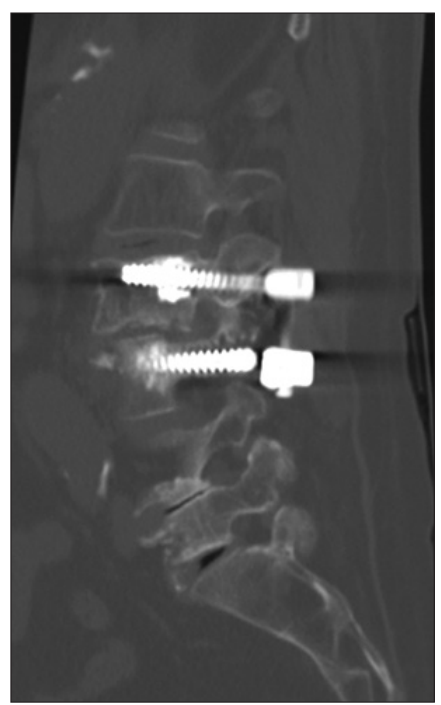

Fig. 4. Breakage of the screw neck in fenestrated screw augmentation.

al. ${ }^{17)}$; the II-F method was superior in a study by Choma et al. ${ }^{18)}$; and there was no difference between the methods in a study by Becker et al. ${ }^{19)}$ In the II-S method, the fixation strength is strongly influenced by the distribution of PMMA. If PMMA mass is located near the tip of a screw, the pullout strength will not increase significantly compared to the use of a conventional unaugmented screw. ${ }^{10,14)}$ The measured values of strength are variable with a wide range because it is not easy to control the distribution of PMMA constantly. On the other hand, in the II-F method, the fixation strength is proportionally influenced by the number of fenestrations. ${ }^{11)}$ In the studies that reported the inferiority of the II-F method, the screws used had either 
Kim et al. Pedicle Screw Augmentation with Bone Cement

Clinics in Orthopedic Surgery • Vol. 12, No. 2, 2020 • www.ecios.org

one ${ }^{17)}$ or two fenestrations. ${ }^{11)}$ The most serious problem with the II-F method is vertebral body fractures that can occur when an augmented screw is pulled out, ${ }^{10,20,21)}$ which could also result in neurological damage. The II-S group in our study had a higher rate of screw loosening and it seemed to be related not only to the anterior location of PMMA mass but also to the crack of PMMA mass (Fig. 3). Such a phenomenon has never been reported in previous experimental studies. If a screw is inserted before the instilled PMMA is cured, a crack would be prevented; however, it is impossible to insert several screws with a pack of PMMA because the working time is limited by curing time. We used fenestrated screws that have four holes with a diameter of $2.5 \mathrm{~mm}$. Screw loosening occurred significantly less in the II-F group than in the II-S group. Loosening occurred between the PMMA mass and the bone, and vertebral body fractures did not occur, contrary to the published experimental data. But screw fractures occurred in three patients all at the neck of the screw (Fig. 4). The fenestrated screws that we used had a diameter of $6.5 \mathrm{~mm}$ or $7.5 \mathrm{~mm}$. All fractured screws were those with a diameter of $6.5 \mathrm{~mm}$. It seemed that the $6.5 \mathrm{~mm}$ screw with a $2.5-\mathrm{mm}$ diameter canula did not have enough strength. But if we had used a smaller canula screw, the risk of leakage would have increased. Leakage of PMMA developed in $4.8 \%$ of the II-S group and in $9.3 \%$ of the II-F group. Compared to the $17 \%$ leakage with use of high-viscosity PMMA in a study by Leichtle et $\mathrm{al}^{14)}$ our results were in an acceptable range and there was no leakage-related complication. Though the difference did not reach statistical significance, it suggests that the II-F method may have a higher risk of leakage because fenestrations of a screw are placed central to the posterior part of a vertebral body. The risk would increase in a fractured vertebra because the position of PMMA instillation could not be controlled according to the pattern of a fracture. In our patients, four of five cases of leakage developed in a fractured vertebra and the cement leaked into the spinal canal in two cases.

There are several limitations in the current study. The number of patients was not enough to prove the statistical difference in the subgroup analysis. The II-S and II-F methods were not used during the same period. However, because the augmentation method was not chosen according to the condition of each patient, the groups and subgroups were thought to be more homogenous. The volume of instilled PMMA is an important factor. The optimal volume was reported as $0.5-3 \mathrm{~mL}$ according to the level of a vertebra. ${ }^{22,23)}$ We instilled 1.5-3.6 $\mathrm{mL}$ of PMMA discretionally to augment a screw. Though we did not analyze the individual volume of PMMA, we do not think the results were influenced by it. We instilled enough volume of PMMA according to the level and size of the individual vertebra. We did not consider the diameter and length of a screw, direction of insertion, and shape of threads of a screw. But the pullout strength of a screw in an osteoporotic vertebra is less influenced by the above variables than a normal vertebra. ${ }^{9)}$

In conclusions, PMMA augmentation of a pedicle screw decreased the incidence of screw loosening in an osteoporotic population. PMMA instillation through screw fenestrations was more effective than prefilling of PMMA with vertebroplasty before screw insertion. However, careful attention and further investigation are necessary to avoid the risk of PMMA leakage and screw fractures with the fenestrated screw augmentation method.

\section{CONFLICT OF INTEREST}

No potential conflict of interest relevant to this article was reported.

\section{REFERENCES}

1. Christodoulou E, Chinthakunta S, Reddy D, et al. Axial pullout strength comparison of different screw designs: fenestrated screw, dual outer diameter screw and standard pedicle screw. Scoliosis. 2015;10:15.

2. Elder BD, Lo SF, Holmes C, et al. The biomechanics of pedicle screw augmentation with cement. Spine J. 2015;15(6):143245 .

3. Frankel BM, D’Agostino S, Wang C. A biomechanical cadaveric analysis of polymethylmethacrylate-augmented pedicle screw fixation. J Neurosurg Spine. 2007;7(1):47-53.

4. Liu D, Shi L, Lei W, et al. Biomechanical comparison of expansive pedicle screw and polymethylmethacrylateaugmented pedicle screw in osteoporotic synthetic bone in primary implantation: an experimental study. Clin Spine Surg. 2016;29(7):E351-7.

5. Liu D, Wu ZX, Pan XM, et al. Biomechanical comparison of different techniques in primary spinal surgery in osteoporotic cadaveric lumbar vertebrae: expansive pedicle screw versus polymethylmethacrylate-augmented pedicle screw. Arch Orthop Trauma Surg. 2011;131(9):1227-32.

6. Kreiner DS, Hwang SW, Easa JE, et al. An evidence-based clinical guideline for the diagnosis and treatment of lumbar 
Kim et al. Pedicle Screw Augmentation with Bone Cement

Clinics in Orthopedic Surgery • Vol. 12, No. 2, 2020 • www.ecios.org

disc herniation with radiculopathy. Spine J. 2014;14(1):18091.

7. Wittenberg RH, Lee KS, Shea M, White AA 3rd, Hayes WC. Effect of screw diameter, insertion technique, and bone cement augmentation of pedicular screw fixation strength. Clin Orthop Relat Res. 1993;(296):278-87.

8. Yi S, Rim DC, Park SW, Murovic JA, Lim J, Park J. Biomechanical comparisons of pull out strengths after pedicle screw augmentation with hydroxyapatite, calcium phosphate, or polymethylmethacrylate in the cadaveric spine. World Neurosurg. 2015;83(6):976-81.

9. Bullmann V, Liljenqvist UR, Rodl R, Schulte TL. Pedicle screw augmentation from a biomechanical perspective. Der Orthopade. 2010;39(7):673-8.

10. Charles YP, Pelletier H, Hydier P, et al. Pullout characteristics of percutaneous pedicle screws with different cement augmentation methods in elderly spines: an in vitro biomechanical study. Orthop Traumatol Surg Res. 2015;101(3):36974.

11. Chen LH, Tai CL, Lai PL, et al. Pullout strength for cannulated pedicle screws with bone cement augmentation in severely osteoporotic bone: influences of radial hole and pilot hole tapping. Clin Biomech (Bristol, Avon). 2009;24(8):6138.

12. Guler UO, Derincek A, Hersekli MA, Ozalay M, Cinar BM, Acaroglu E. Restoration of pull-out strength of the failed pedicle screw: biomechanical comparison of calcium sulfate vs polymethylmethacrylate augmentation. Acta Orthop Traumatol Turc. 2014;48(2):202-6.

13. Kang SH, Cho YJ, Kim YB, Park SW. Pullout strength after expandable polymethylmethacrylate transpedicular screw augmentation for pedicle screw loosening. J Korean Neurosurg Soc. 2015;57(4):229-34.

14. Leichtle CI, Lorenz A, Rothstock S, et al. Pull-out strength of cemented solid versus fenestrated pedicle screws in osteoporotic vertebrae. Bone Joint Res. 2016;5(9):419-26.
15. Tolunay T, Basgul C, Demir T, Yaman ME, Arslan AK. Pullout performance comparison of pedicle screws based on cement application and design parameters. Proc Inst Mech Eng H. 2015;229(11):786-93.

16. Cook SD, Salkeld SL, Stanley T, Faciane A, Miller SD. Biomechanical study of pedicle screw fixation in severely osteoporotic bone. Spine J. 2004;4(4):402-8.

17. Chang MC, Kao HC, Ying SH, Liu CL. Polymethylmethacrylate augmentation of cannulated pedicle screws for fixation in osteoporotic spines and comparison of its clinical results and biomechanical characteristics with the needle injection method. J Spinal Disord Tech. 2013;26(6):305-15.

18. Choma TJ, Pfeiffer FM, Swope RW, Hirner JP. Pedicle screw design and cement augmentation in osteoporotic vertebrae: effects of fenestrations and cement viscosity on fixation and extraction. Spine (Phila Pa 1976). 2012;37(26):E1628-32.

19. Becker S, Chavanne A, Spitaler R, et al. Assessment of different screw augmentation techniques and screw designs in osteoporotic spines. Eur Spine J. 2008;17(11):1462-9.

20. Costa F, Ortolina A, Galbusera F, et al. Pedicle screw cement augmentation: a mechanical pullout study on different cement augmentation techniques. Med Eng Phys. 2016;38(2):181-6.

21. Bullmann V, Schmoelz W, Richter M, Grathwohl C, Schulte TL. Revision of cannulated and perforated cement-augmented pedicle screws: a biomechanical study in human cadavers. Spine (Phila Pa 1976). 2010;35(19):E932-9.

22. Derincek A, Wu C, Mehbod A, Transfeldt EE. Biomechanical comparison of anatomic trajectory pedicle screw versus injectable calcium sulfate graft-augmented pedicle screw for salvage in cadaveric thoracic bone. J Spinal Disord Tech. 2006;19(4):286-91.

23. Aydogan M, Ozturk C, Karatoprak O, Tezer M, Aksu N, Hamzaoglu A. The pedicle screw fixation with vertebroplasty augmentation in the surgical treatment of the severe osteoporotic spines. J Spinal Disord Tech. 2009;22(6):444-7. 